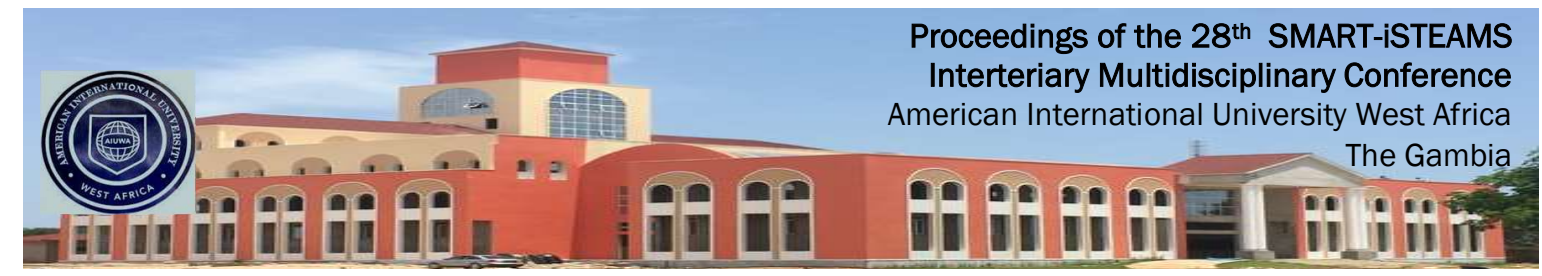

Full Research Paper

\title{
A Study of Energy Efficiency in Wireless Sensor Networks
}

1Benjamin Panful

2Xie Zhibin

American International University West Africa

The Gambia

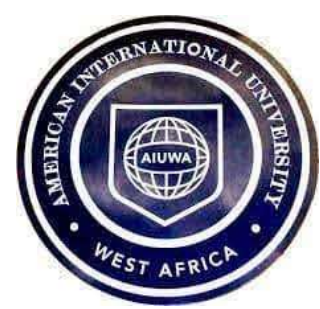

2Jiangsu University of Science \& Technology, China

E-mails benpanful@yahoo.com xiezhibin@just.edu.cn

Phones

$+2202571036$

$+8613775551797$

\section{ABSTRACT}

The wireless sensor networks (WSNs) field is one of the emerging and fast-growing fields in the scientific world. This has brought about the development of low-cost, and multifunctional sensor nodes for easy and secure dissemination of information. Nonetheless, the problem of sensor nodes running out of energy quickly has been an issue. Many energyefficient routing algorithms have been proposed to solve this problem and preserve the longevity of the network. Most of the recent papers have shown so many protocols mainly designed to minimize energy consumption in the network but there are some limitations with regards to the area of operation and the optimization decisions. This paper proposes an Energy Efficiency Hierarchical Routing Technique (EEHRT) which is based on LEACH protocol to extend the lifespan of a WSN. In this work, the proposed Energy Efficient Hierarchical Routing Technique selects cluster heads based on the prediction of transmission energy through the shortest distance to the base station. Our approach rotates the role of Cluster Heads $(\mathrm{CHs})$, and optimizes the $\mathrm{CH}$ selection by the prediction of energy transmission in every round and aggregating data before transmission to the BS. The important features which include member nodes formation and rotation, cluster head selection and rotation, and cluster optimization of our proposed hierarchical routing technique in transmitting data to the base station are analyzed and emphasized.

Keywords: Wireless Sensor Network, Base Station, Member Node, Clustering, LEACH, Hierarchy

Proceedings Reference Format

Benjamin Panful \& Xie Zhibin (2021): Rural Women's Access to Land and its Implications for Empowerment in Nigeria: The Case of Ilorin East Local Government Area, Kwara State, Nigeria. Proceedings of the 28th iSTEAMS Intertertiary Multidisciplinary Conference. American International University West Africa, The Gambia. Series 28, Vol 3 October 2021. Pp 105-120 www.isteams.net/gambia2021.

DOI - https://doi.org/ 10.22624/AIMS/iSTEAMS-2021/V28N3P9 


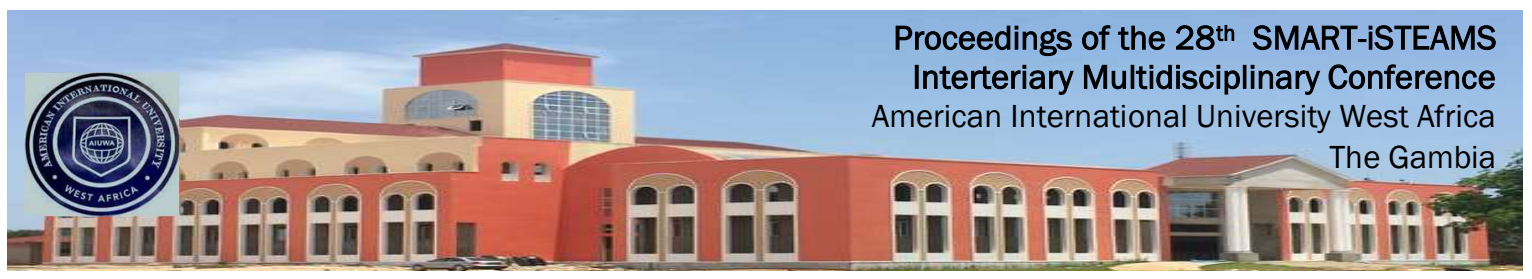

\section{INTRODUCTION}

\subsection{Background}

A wireless sensor network (WSN) is a wireless network that contains independent devices with sensors that are spread over a specific area to supportively observe conditions such as vibration, pressure, sound, temperature, motion contaminants at different positions in the environment[1]. WSN can contain hundreds or thousands of low-powered multi-functioning nodes, which work in an unattended environment with restricted computing and sensing functions[2]. The nodes in WSN are normally equipped with some radio transceivers, tiny microcontrollers and some batteries which provide energy to the nodes[3]. The network for monitoring a specific area in an environment is formed by these low-powered and inexpensive sensor nodes. The WSNs gather and transmit various kinds of data such pressure, sound and temperature about the monitored surrounding through the co-operation of the sensor nodes. The WSN involves a sizable number of sensor nodes with limited energy source which operate without any central infrastructure and a control point. The sensor nodes gather data locally from the environment and then forward them to the specific site known as Base Station (BS)[4].

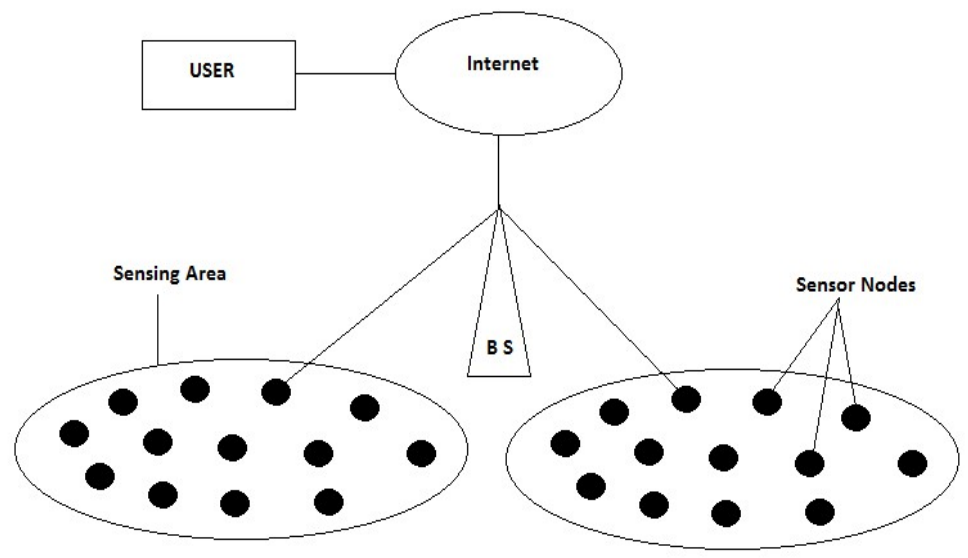

Figure 1.1: Components of Wireless Sensor Network (WSN)

When a large number of sensor nodes are deployed in a large area to co-operatively monitor a physical environment, the networking of these sensor nodes is equally important. A sensor node in a WSN does not only communicate with other sensor nodes but also with a Base Station (BS) using wireless communication[5].From Figure1.1 the base station sends commands to the sensor nodes and the sensor node perform the task by collaborating with each other. After collecting the necessary data, the sensor nodes send the data back to the base station.The base station also acts as a gateway to other networks through the internet. After receiving the data from the sensor nodes, a base station performs simple data processing and sends the updated information to the user via the internet[6]. Recently, there has been a huge development in the micro-electromechanical systems (MEMS) based technologies and this has brought about the deployment of large number of small sensor nodes. 


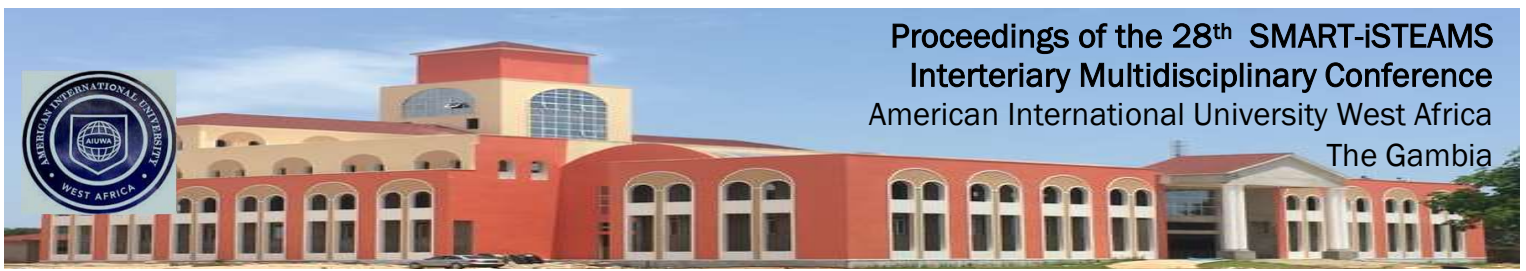

The energy of sensor nodes has a major role in Wireless Sensor Networks[7]. Mostly the network failure happens because of lack of energy in the sensor nodes. The lifetime of sensor nodes is limited. So the WSN should efficiently use the sensor nodes and the power or energy and also the throughput should be efficient[8].There are number of algorithms and protocols which have been proposed to reduce the energy consumption in WSN. From those algorithms, few are well suitable solution for the wireless sensor networks but for this research, we will study and evaluate the effectiveness of Low-Energy Adaptive Clustering Hierarchy (LEACH) protocol in cluster head selection, and proposes an improved clustering algorithm in the subsequent chapters[9].

The idea and the development of WSNs were initially popularized in the military through the application of battlefield sound surveillance systems to detect and trace the enemies' submarines. Nowadays, the application of this technology is not only seen in the military and the national security fields. The technology has however been made available in a wide range of areas such as the agricultural, environmental monitoring and many other fields[10]. WSN is growing rapidly in our modern society. Smart homes which are characterized by the wireless connection of controllable and automated devices which constantly monitor the homes, industrial locations, organization settings and the general environment are all based on the WSN technology[11]. Energy consumption has been a huge concern throughout the development of WSNs. The main source of energy of the sensor nodes in the WSN is small batteries[12]. For this reason, any technique to optimize the energy usage in WSN would be of great benefit to increase the lifespan of the network.

1.2 Aims and Objectives

The main objective of this paper is to improve upon the Low-Energy Adaptive Clustering Hierarchy (LEACH) protocol and propose an optimized algorithm for the clustering to prolong the network's lifetime.To achieve this goal, we have proposed a technique whose principle of cluster head selection is based on the forecast of the highest residual energy after the subsequent round and the shortest distance through the nearest adjoining cluster head to the BS. Increasing the network's lifespan is a great way of making the network more efficient. LEACH occupies more position in the field of WSN and most hierarchical routing protocols, which seek to extend the lifetime of networks are usually based on $\operatorname{LEACH}[13,14]$. In the area of WSNs, Communication, Sensing and Computation are the three major aspects that consume a lot of energy.

When designing WSNs, the communication parameter should be made a primary objective whereas the sensing and the computation parameters could be made a secondary objective[15]. The communication cost is higher that of sensing and computation. Therefore, any efforts to reduce the communication cost by the right cluster head will go a long way to increase the lifespan of the network. Cluster heads in hierarchical routing protocols consume more energy since they are responsible for data collection and internal and external communications of the cluster[16].The secondary objective of this work is to achieve a network with an optimum number of cluster heads at each round of data transmission. By the end of this work, a network with an optimized number of cluster heads would be realized. 


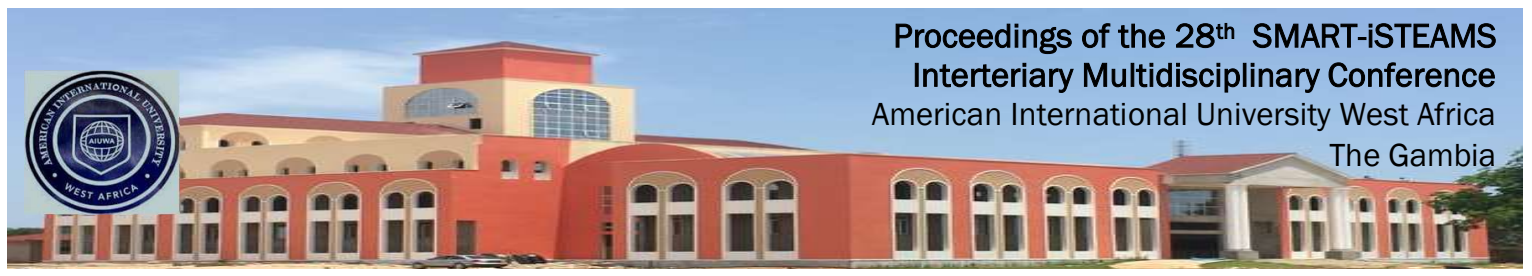

\subsection{Significance of the Study}

The design of WSNs has two main challenging objectives. One is the network's ability to transmit a large amount of data between the BS and the Sensor Nodes (SN)[17]. The other challenge is the minimization of energy consumption[18]. These two constraints show the significance of efficient routing algorithms in the design of WSNs. Because of the challenges in the design of efficient WSNs, many routing protocols have been proposed. Of all those techniques proposed, hierarchical routing algorithms highly satisfy the shortcomings in the WSNs design[19]. A hierarchical routing algorithm is usually understood to be a double layer architecture, where one layer mustselect the cluster head $(\mathrm{CH})$ and the other layer is tasked with routing responsibility[20]. In a hierarchical routing protocol, a cluster head is the node with is responsible for data collection from other nodes in the cluster, accumulating and compressing all the data and then transmitting the data to the Base Station (BS)[21].

In this work, the LEACH protocol is analyzed. Our analysis of LEACH leads to the development of a new and more energy-efficient routing technique. It is important to note that, graphical representation of data to visualize the network behavior is important when working with a large amount of varying data. Energy consumption is crucial in this work so the energy level of every node in the network is of great interest.

\section{LITERATURE REVIEW}

\subsection{The Concept of Clustering}

Hierarchical routing technique is mostly employed to transmit data from many sensor nodes towards one BS in a more efficient way[22]. The purpose of cluster-based routing is to make use of a network routing hierarchy that is based on a sizeable number of clusters as indicated in Figure 2.1 below.

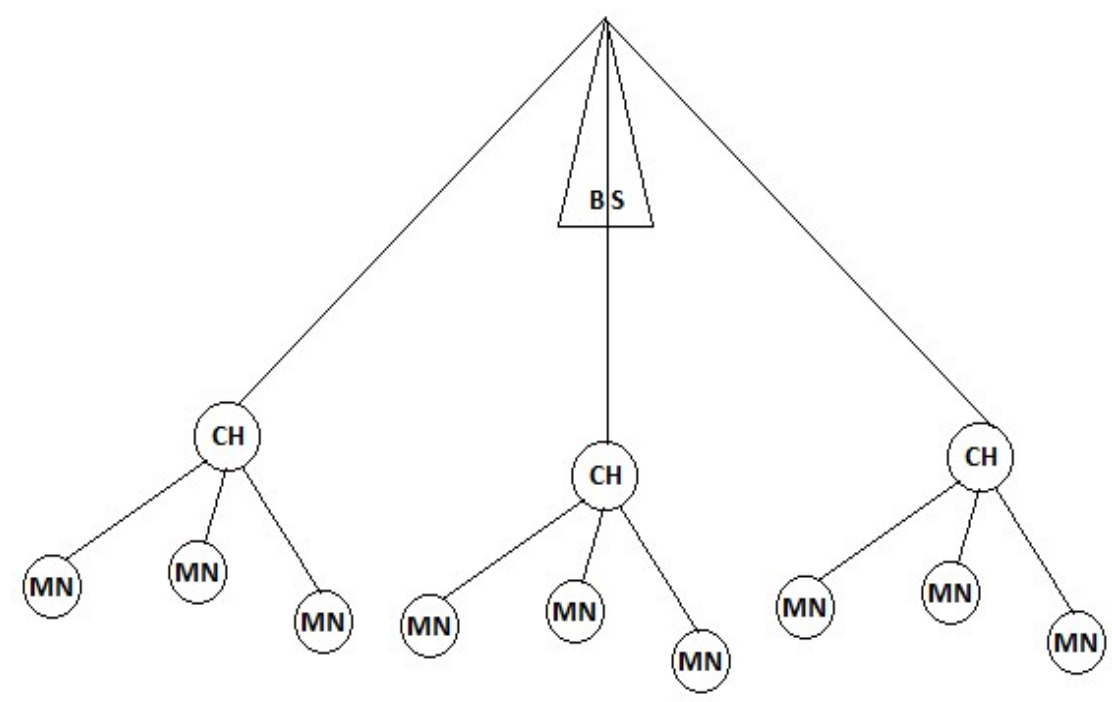

Figure 2.1 A Clustered network 


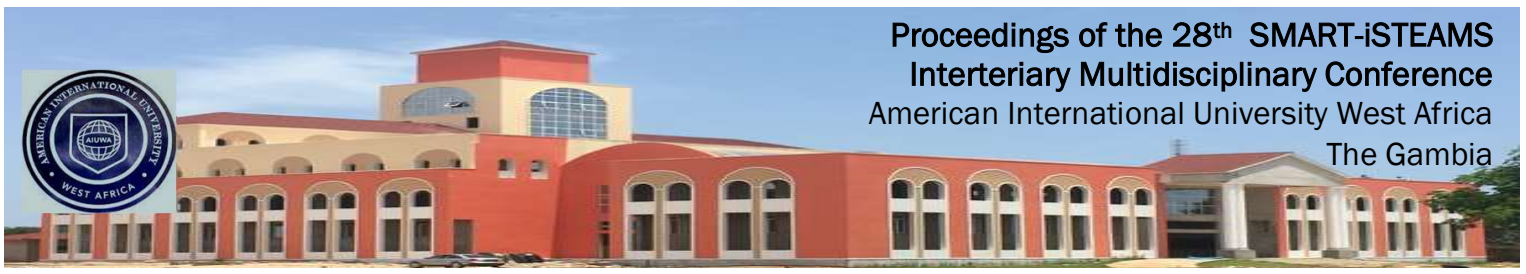

Clustering is the method of grouping sensor nodes into small-sized clusters with the aim of reducing the data transmission time and the energy consumption rate of the sensor nodes in the WSN[23]. As shown in figure 2.1 some nodes are selected as Cluster Heads (CHs) based on a particular selection method and the remaining nodes are termed as Member Nodes(MNs). The MNs are responsible for collecting environmental data and transmitting them to the $\mathrm{CHs}$. The CHs aggregates and further send the data to the Base Station (BS)[24].

To deal with the problem of scalability, sensor nodes can be grouped in clusters in order to simplify the mode of communication between them[25]. Every cluster has a unique node called Cluster Head $(\mathrm{CH})$ in addition to some Member Nodes (MNs). The $\mathrm{CH}$ must gather data from the MNs in the respective cluster. In a clustered network, the data of each node is transmitted locally in its matching cluster but not transmitted through the entire network[26]. Every $\mathrm{CH}$ can recognize and receive data from their $\mathrm{MNs}$ in their corresponding cluster and other $\mathrm{CHs}$ that could be used to relay data to the BS. However, each MN has knowledge about only its $\mathrm{CH}$ but not about other nodes in the whole network. This makes the whole network looks smaller in the sight of all the nodes in the network.

\subsection{The Benefits of Clustering}

The sensed data in a cluster can have some form of correlation since the MNs are mostly sited close to each other. It is therefore imperative for the $\mathrm{CH}$ to perform data aggregation in order to remove redundant data by using suppression, maximum, minimum and average functions. This brings distinctions between raw and useful data[27]. For example, if MNs are sensing and transmitting data, the matching $\mathrm{CH}$ can determine the average value of the data and relay it as a single message. That is, data aggregation decreases the amount of data that is relayed towards the BS as well as the number of data transmissions in the entire network. This goes a long way to contribute to the better utilization of the limited bandwidth. Knowing that the amount of energy required in data computation is usually smaller than that used in data transmission, data aggregation can also reduce the overall energy consumption in the network and increase the network's lifespan[28].

In a clustered network, the number of computations required to determine the routing paths through which data is delivered to the BS is reduced[29]. This is because only the $\mathrm{CH}$ does the routing so there is less exchange of routing data among the nodes in the network. Also, the size of the routing overhead and the routing tables are reduced. This helps to minimize the network's energy consumption and further increases the network's lifespan. In flat routing protocols, all the nodes in the network have the same duties in the network and data usually through many hope and nodes before finally arriving at the BS[30]. This situation can increase the data transmission time. However, in hierarchical routing methods, data covers a bigger distance as it moves from one level to another .here, the data transmission rate is faster and the latency can be reduced[31]. 


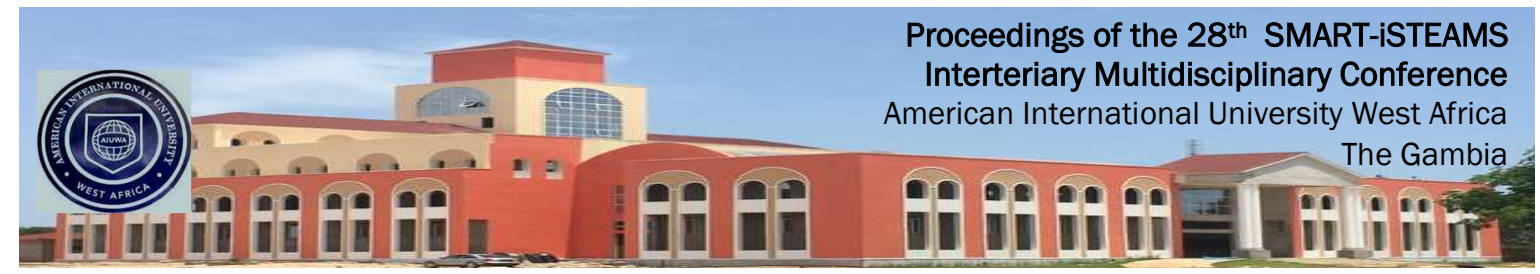

From the above discussion, it could be seen that clustering routing techniques have some better advantages over the flat routing techniques. It is however pertinent to note that, clustering routing techniques also have some drawbacks such as the overhead that is likely to be incurred as a result of the exchange of messages among the nodes in the network during the cluster configuration and maintenance processes[32]. In all, the benefits of clustering routing techniques overshadow its limitations concerning energy consumption.

2.3 The Elements Examined in the Design of Cluster-Based Algorithms

A cluster-based protocol could either be implemented in a centralized or a distributed style[33].No central point is required to execute a distributed algorithm. The network configuration job is shared among all the member nodes in the network. The member nodes rely on the local information available to them to make network configuration decisions. This method is helpful because it does not keep any global information about the network. Nonetheless, the network configuration decision process by the member nodes requires some amount of computational resources.

With the centralized protocol, the BS is used as a central point to configure the network[34]. The BS does not usually have limited computation and energy issues, so it is mostly used to execute the tasks that need a high amount of energy. The use of a centralized protocol can offer better control of the routing in the network. In either the centralized or the distributed clustering algorithm, there are three key aspects to be considered namely the

- cluster head selection

- cluster forming

- cluster communications

\subsubsection{Cluster Head Selection}

In WSN, a $\mathrm{CH}$ could be a node with more resources than a regular node. In the case of $\mathrm{CHs}$ being a regular node, it is pertinent for re-clustering to be done to select a new $\mathrm{CH}$ [35]. This is done to avoid the quick depletion of the CH's energy that may occur due to the various tasks it performs. CHs re-selection can also be a means of fault tolerance. Reselecting a new $\mathrm{CH}$ helps in establishing a new communication link for MNs when an old $\mathrm{CH}$ dies. When selecting a $\mathrm{CH}$, it is important to consider the node's location and residual energy[36]. The amount of CHs selected has an effect on the overall energy dissipation of the network. When the selected CHs are few, the distance between the MNs and their respective $\mathrm{CHs}$ is likely to be far apart. This situation increases the energy consumed in transmitting the data from the MNs to their corresponding $\mathrm{CHs}$.

A network with too many $\mathrm{CHs}$ which are closely located could result in collisions and redundancy in their data transmission especially when the data is assumed to be correlated[37]. Again, a $\mathrm{CH}$ consumes more energy than MN due to do the kind of tasks it performs, therefore have too many $\mathrm{CHs}$ in the network tends to increase the overall energy dissipation in the entire network[38]. From the discussion above, it is essential to consider the number of $\mathrm{CHs}$ to be chosen in the network for optimized energy utilization. 


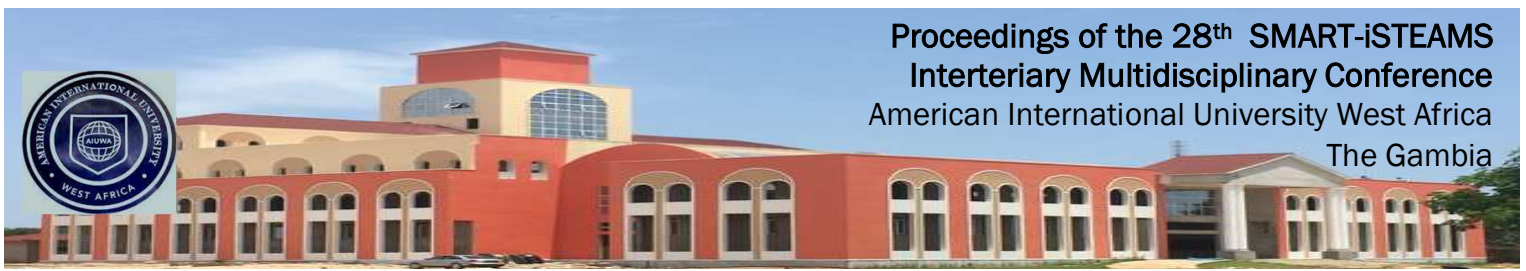

\subsubsection{Cluster Formation}

In cluster formation, the MNs of every selected $\mathrm{CH}$ s are identified to form various clusters. When clusters are being formed, it is important to make sure that MNs do not consume a large amount of energy whiles transmitting data to their respective $\mathrm{CHs}[39,40]$.The duties of the MNs contribute to the burden of their $\mathrm{CHs}$ since every $\mathrm{CH}$ has to collect and aggregate the data from its NMs and later forward them to the BS. This implies that the more the tasks of the MNs increase, the more their corresponding $\mathrm{CH}$ s consume more energy. When clusters are formed in such a way that they have a similar sized and balanced load, it helps the $\mathrm{CH}$ to use less energy in performing their tasks.

\subsubsection{Cluster Communication}

Intra-cluster communication and inter-cluster communication are the two main types of cluster communications[41]. With the intra-cluster communications, the data exchanges occur between each of the MNs and their respective $\mathrm{CHs}$. This type of communication normally includes a one-hop transmission between each of the MNs and their $\mathrm{CH}$ shich simplifies the communications. Multi-hop transmissions become beneficial in cases, where the MNs have a very short transmission range and cannot reach their corresponding $\mathrm{CHs}$.

With inter-cluster communications, the interaction is normally between the $\mathrm{CH}$ and the $\mathrm{BS}[42]$. This type of communication typically determines the path between each of the $\mathrm{CH}$ and the $\mathrm{BS}$ to transmit data. In small networks where the $\mathrm{CHs}$ are closer to the BS, a single hop becomes beneficial in transmitting data from the $\mathrm{CH}$ s to the $\mathrm{BS}$. When the distance between the $\mathrm{CHs}$ and the BS are far apart, multi-hop transmission becomes more prudent in relaying data from the $\mathrm{CHs}$ to the BS.

\section{THE PROPOSED ROUTING TECHNIQUE}

\subsection{Our views on the existing cluster-based algorithms}

In centralized protocols, the BS exploits its global knowledge of the network to produce better decisions, as opposed to distributed protocols where decisions are made locally and may not be the most efficient $[43,44]$. This can help in avoiding uncertainty at the nodes since the BS is making decisions on their behalf while taking into consideration the conditions in the entire network. Utilizing a centralized algorithm can provide robustness where the BS reacts to any changes in the nodes' conditions by re-configuring the network and providing the new routing paths. Moreover, centralizing the algorithm can offer flexibility in computing the entire routes as opposed to determining only the next-hop at the nodes themselves which is the case in distributed algorithms[45, 46].

In centralized algorithms, the required computations are shifted from the nodes, which have limited computation capability and energy supply, to the BS, which has a much higher computation capability and no energy limitation[47, 48]. Having a centralized algorithm can avoid the need for exchanging messages between the nodes in the association process, e.g., nodes announcing they are $\mathrm{CHs}$, and nodes choosing and joining their $\mathrm{CHs}$, which are used in distributed algorithms. This can reduce the amount of signaling messages, helping to reduce collisions in the network. 


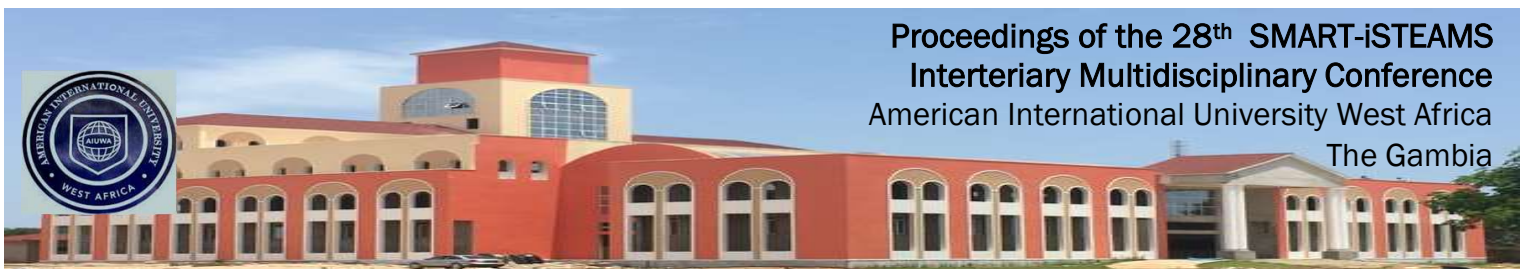

However, centralized algorithms still require exchanging messages between each of the nodes and the BS, which needs to be considered in designing a centralized cluster-based algorithm[49, 50].

Selecting $\mathrm{CHs}$ in a deterministic manner can better control the decision and can produce better distributed $\mathrm{CHs}$. This is different from employing a probability function where nodes make the decision independently like in LEACH .Employing the residual energy as a parameter in $\mathrm{CH}$ selection can ensure that only nodes with high remaining energy are elected as $\mathrm{CHs}$ [51]. This can balance the energy consumption among the nodes. For cluster forming, LEACH-C considers only the distance parameter in determining the $\mathrm{MNs}$ of each $\mathrm{CH}$. This may result in unbalanced cluster sizes, unbalanced energy consumption among $\mathrm{CHs}$ and thus, cannot effectively extend network lifetime.

In many of the existing cluster-based protocols, $\mathrm{CH}$ s deliver their data to the BS in a single hop manner, like in LEACH-C[52]. This method requires high transmission power when $\mathrm{CH}$ are far away from the BS. To address this issue, other protocols construct multi-hop routing paths between $\mathrm{CHs}$ and the BS based on residual energy alone or distance alone[53]. However, forwarding data based on the residual energy of the next-hop, $\mathrm{CH}$ alone can result in long-range transmissions which will require high transmission power. On the other hand, considering only the distance to the nexthop, the $\mathrm{CH}$ 's energy can be depleted quickly. Relying on one $\mathrm{CH}$ to relay data to the BS depletes its energy faster than the other $\mathrm{CHs}$, which results in unbalanced energy consumption among $\mathrm{CHs}$. Unbalanced load results in unbalanced energy consumption, which shortens the network's lifetime[54]. This is one of the issues that need to be considered in the case of multi-hop inter-cluster communication.

Furthermore, in the existing centralized cluster-based algorithms, all the nodes in the network send their status information in every round to the BS[55]. This information is used in configuring the network. However, in practice, the network might suffer from a large amount of traffic because of these transmissions. Also, some of these transmissions can be redundant since the change in energy may not be significant and therefore, does not affect the network configuration. Unnecessary transmissions of nodes information increase the total energy consumption. That is another issue to be considered.

More importantly, the existing cluster-based algorithms employ TDMA[56] schedules and CDMA codes for data transmission between MNs, CHs, and the BS[57]. In practice, it is hard to create such schedules and codes since they require synchronization between the nodes in the network, which is difficult to achieve in ad-hoc WSNs. For this reason, many WSNs do not use TDMA or CDMA schemes. Instead, they employ CSMA for the simplicity of access control. That is, it is asynchronous and the transmission can be done in a distributed manner. This means that there is no need to assign a time slot for each node that needs to transmit data. Again, the main standard for WSNs is IEEE 802:15:4 which employs CSMA for data transmission[58]. Therefore, it is more practical to have a routing algorithm that utilizes only CSMA during data transmission. 


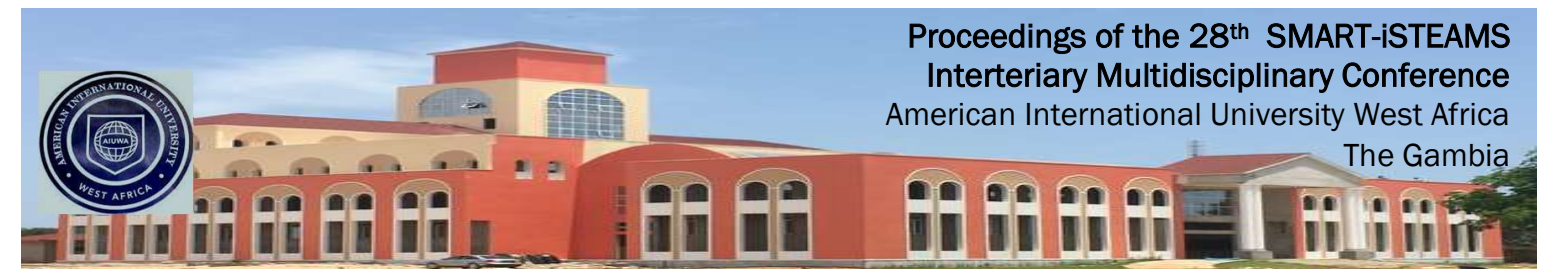

\subsection{System Energy Model of a Sensor Node}

Having an understanding that the wireless communication component of a sensor node is responsible for the energy-draining activities, we use the radio model shown in Figure 3.1. The first order radio model offers an evaluation of energy consumed when transmission or reception is made by a sensor node at each cycle[59]. The radio has power control to expend the minimum energy required to reach the intended recipients.

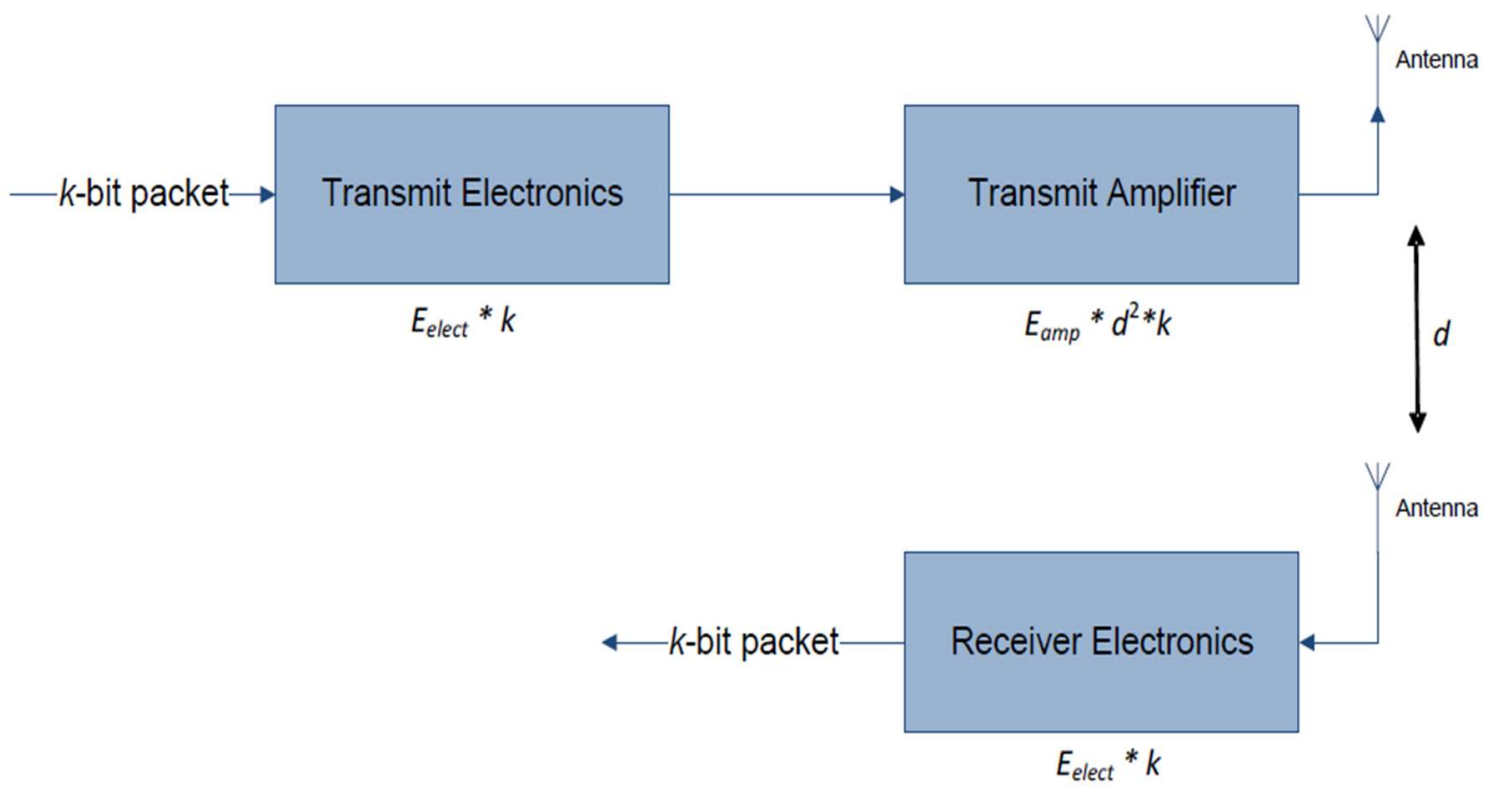

Figure 3.1: Schematic diagram of the first order radio model

Mathematically, when a $k$-bit message is transmitted through a distance, $d$, required energy can be expressed as stated in the equation:

$E_{T x}=E_{\text {elect }} \cdot k+E_{a m p} \cdot d^{2} \cdot k$

Similarly, the energy consumed at the reception is shown in the equation below:

$E_{R x}=E_{\text {elect }} \cdot k$

Where:

$E_{T x-e l e c t}$ is the energy dissipation per bit at the transmitter.

$E_{R x \text {-elect }}$ is the energy dissipation per bit at the receiver.

$E_{a m p}$ is the amplification factor

$E_{\text {elect }}$ is the cost of circuit energy when transmitting or receiving one bit of data

$k$ is the number of transmitted data bit

$d$ is the distance between a sensor node and its respective cluster head 


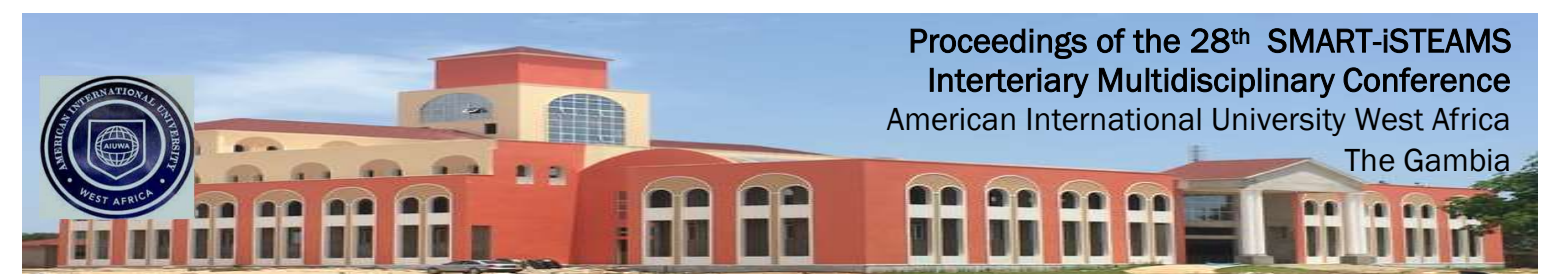

The first order radio equation was used to verify the operation of our proposed algorithm, assuming that the radio channel being symmetric such that equal energy is used up when a node $A$ transmits to node $B$ and when a node $B$ transmits to node $A$ with a given signal to noise ratio.

\subsection{Our proposed technique}

Based on our views expressed in sub-section 3.1 regarding the issues in the existing clusterbased algorithms, we have proposed an Energy Efficient Hierarchical Routing Technique (EEHRT) for WSN. The main objective of our proposed technique is to reduce the total energy consumption in the network and to extend the network'slifetime. Our proposed technique is based on the principle of LEACH protocol.To minimize the energy consumption in WSNs, we have proposed a technique whose principle of cluster head election is based on the forecast of the highest remaining energy after the subsequent round and the shortest distance through the nearest cluster head to the BS. Since hierarchical routing technique offers a better scalability and efficient communication approach, its concept can also be effectively used to design energy efficient routing algorithm in WSN.

In the proposed model, cluster formation is based on equal division of the area. Apart from the one cluster formation which makes use of the entire sensors area, other formation such as two clusters formation and three clusters formation involves equal segregation of the area. The Cluster Head selection stage comes after the cluster formation stage. The selection of $\mathrm{CH}$ within each cluster is done by selecting a node that requires less transmission energy to be the $\mathrm{CH}$ for a particular transmission round. Due to draining activities being constraint on a cluster head during data aggregation and transfer phase, the cluster head is rotated among the sensor nodes of each cluster at every transmission round. A completely new estimation of energy is carried out at the beginning of every transmission round to elect a new $\mathrm{CH}$ for the cluster therefore energy wastage is being reduce to its minimum, and utilization of each node's energy is being maximized to ensure an increased network lifetime.

The technique consists of following steps:

i. Cluster formation which is based on equal division of the area .

ii. Election of $\mathrm{CHs}$

iii. Data aggregation

iv. Data transmission

The Cluster Head election can be also be described in the following four stages:

i. The initial energy $E_{\text {in }}(n)$ of the node is measured

ii. The distance $d(n)$ from each node to the BS or/to the corresponding higher level $\mathrm{CH}$ is measured

iii. Estimation of the energy required by each node for transmission within the cluster is carried out using the formula: $\left(E_{a m p} * k * d^{2}\right)$

iv. The maximum energy after the subsequent transmission round for each node is estimated and selection of $\mathrm{CH}$ is done using the formula:

$\max \left(E_{\text {in }}(n)-E_{\text {amp }} * k * d^{2}\right)$ then after the $\mathrm{CH}$ selection is carried out, the next cluster head selection will take place after the current round is completed. 


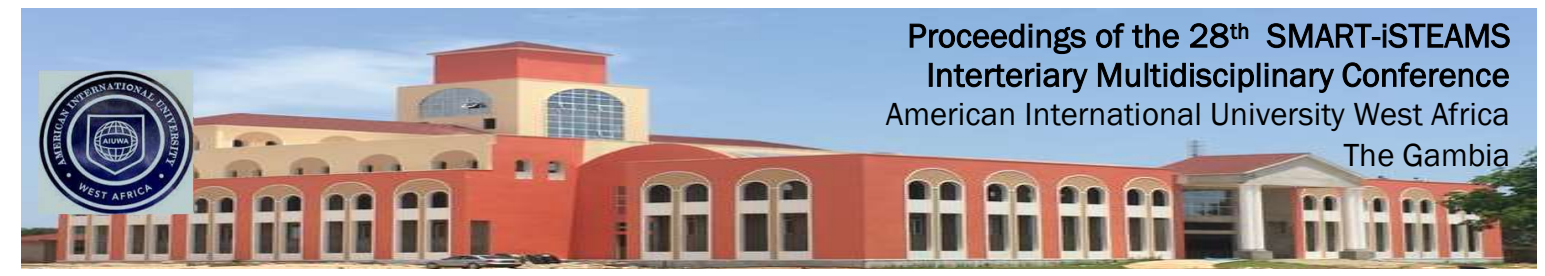

A sleep-awake technique could be employed to avoid energy loss in the network. The maximum distance node is checked in each round after which the energy that is needed for data transmission is calculated $\left(E_{t h}\right)$. In each round, if the energy level of a node $\left(E_{n}\right)$ is less than or equal to $\left(E_{t h}\right)$, the sensor node can transmit data but if the $\left(E_{n}\right)$ is less than $\left(E_{t h}\right)$, it goes into sleep mode to conserve energy.

The sleeping schedule of each node is set according to the $\left(E_{t h}\right)$. We use the following equation to calculate the $\left(E_{t h}\right)$.

$E_{t h}=((E T X+E D A) * D)+\left(E_{a m p} * D * d^{4}\right)$

Where $D$ is the length of data packet and $d$ is the distance between the maximum distance node and the sink. The $\mathrm{CHs}$ in the network forward both data generated by themselves and that of the MNs. The $\mathrm{CH}$ receives $\left(D_{1}+D_{2}+D_{3}+\ldots+D_{N}\right)$ and sends $\left(D_{C H}+D_{1}+D_{2}+\right.$ $\left.D_{3}+\ldots+D_{N}\right)$. When the distance between the $N$ and $C H$ is $d<d_{0}$, the energy consumption is given in equation (3.4)

$E_{N}^{C H}=D_{N}^{C H}\left(E_{\text {ele }}\right)+D_{N}^{C H}\left(E_{f s}\right)\left(d^{2}\right)$

When the distance between $N$ to $C H$ is $>d_{0}$, the energy consumption is given in equation (3.5)

$E_{N}^{C H}=D_{N}^{C H}\left(E_{\text {ele }}\right)+D_{N}^{C H}\left(E_{\text {amp }}\right)\left(d^{4}\right)$

Energy consumed by $\mathrm{CH}$ to transmit data to the $\operatorname{Sink}(\mathrm{S})$ when the distance between them is $d<d_{0}$ is given in equation (3.6)

$E_{C H}^{S}=D_{C H}^{S}\left(E_{\text {ele }}\right)+E_{D A}+D_{C H}^{S}\left(E_{f S}\right)\left(d^{2}\right)$

When the distance between the $\mathrm{CH}$ and $\mathrm{S}$ is $d>d_{0}$, the energy consumption is given in equation(3.7)

$E_{C H}^{S}=D_{C H}^{S}\left(E_{\text {ele }}\right)+E_{D A}+D_{C H}^{S}\left(E_{a m p}\right)\left(d^{4}\right)$

$E_{\text {Total_CH }}=E_{C H}+E_{N}(3.8)$

$E_{\text {Average_CH }}=\frac{E_{\text {Total_CH }}}{N}$

Energy saving in each round for a normal node:

$E_{\text {Save }_{N}}=E_{\text {ele }}+E_{T X}+E_{\text {amp }}(3.10)$ 


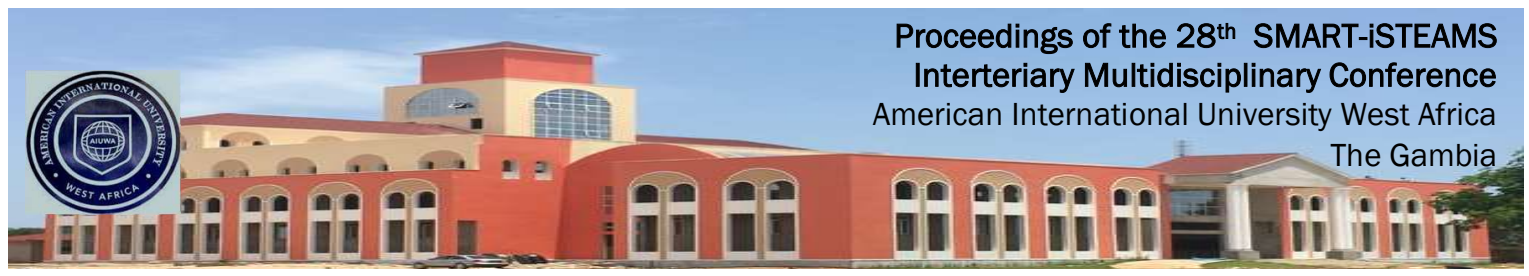

Energy saving for $\mathrm{CH}$ :

$E_{\text {Save_CH }_{-}}=E_{\text {ele }}+E_{D A}+E_{T X+} E_{R X}+E_{a m p}$

Energy saving for all sleep nodes is given in equation (3.12)

$E_{\text {Save }_{\text {Total }}}=\sum_{i=0}^{n} E_{i}$

The sleep-awake technique is based on the $\left(E_{t h}\right)$. The nodes that are far from the sink have a high probability of sleeping, therefore conserving energy to increase the network's lifespan. Due to the limited energy of sensor nodes, the implementation of the sleep-wake technique is very useful to enhance the lifetime of the network.

\section{CONCLUSION}

\subsection{Conclusion}

A major challenge in designing an efficient protocol for wireless sensor networks is maximizing the network's lifetime. Clustering has been considered one of the most viable methods for addressing the challenge of maximizing network lifetime. In this paper, we proposed an energy efficient hierarchical routing technique in which cluster heads are elected based on the prediction of transmission energy via a shortest distance to the base station. Our approach rotates the role of $\mathrm{CH}$, and optimize the $\mathrm{CH}$ selection by prediction of energy transmission in every round and aggregating data before transmission to the BS. The important features which include cluster formation and rotation, cluster head election and rotation, and cluster optimization of our proposed hierarchical routing technique in transmitting data to the base station were emphasized.

The concept of hierarchical routing technique can be effectively used to design energy-efficient routing protocol in WSN. With the energy awareness algorithm being our core interest in this work, our proposed hierarchical technique, which uses the prediction of the smallest transmission energy via the shortest path possible to send data to the BS offers more reduced energy consumption and also increases the lifetime of the WSN. 


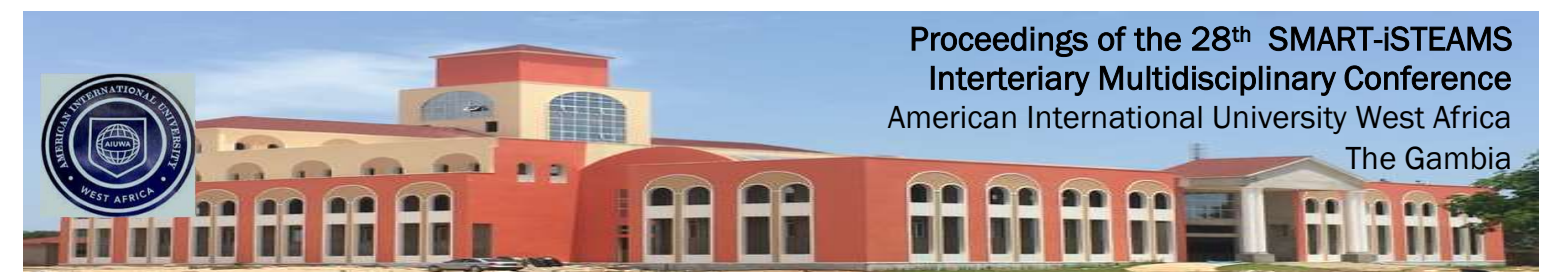

\section{REFERENCES}

[1] V. J. Hodge, S. O'Keefe, M. Weeks, and A. J. I. T. o. I. T. S. Moulds, "Wireless sensor networks for condition monitoring in the railway industry: A survey," vol. 16, no. 3, pp. 1088-1106, 2015.

[2] S. Singh and J. Singh, "Multi-hop Stable Selection with Mobile Sink in Wireless Sensor Network," 2013.

[3] M. Charalampidou, G. Pavlidis, and S. G. J. I. Mouroutsos, "A novel modular wireless sensor networks approach for security applications," vol. 12, no. 1, pp. 40-50, 2017.

[4] G. Z. Papadopoulos, A. Gallais, G. Schreiner, and T. Noël, "Importance of repeatable setups for reproducible experimental results in loT," in Proceedings of the 13th ACM Symposium on Performance Evaluation of Wireless Ad Hoc, Sensor, \& Ubiquitous Networks, 2016, pp. 51-59: ACM.

[5] M. Z. Khan, M. Merabti, B. Askwith, and F. Bouhafs, "A fault-tolerant network management architecture for wireless sensor networks," in 11th Annual PGNet 2010 Conference, At Liverpool John Moores University, UK, 2010.

[6] P. Buonadonna, D. Gay, J. M. Hellerstein, W. Hong, and S. Madden, "TASK: Sensor network in a box," in Proceeedings of the Second European Workshop on Wireless Sensor Networks, 2005., 2005, pp. 133-144: IEEE.

[7] K. Manikandan, P. Kanmani, M. J. I. J. o. A. R. i. C. Sulthana, and C. Engineering, "Energy efficient algorithms for wireless sensor network," vol. 4, no. 1, pp. 342-346, 2015.

[8] B. Bhushan and G. Sahoo, "Routing Protocols in Wireless Sensor Networks," in Computational Intelligence in Sensor Networks: Springer, 2019, pp. 215-248.

[9] B. P. KUMAR, D. KRISHNA, and P. RAVINDRA, "Secure and Efficient Data Transmission for Cluster-Based Wireless Sensor Networks," 2015.

[10] J. Sánchez-García, J. García-Campos, M. Arzamendia, D. Reina, S. Toral, and D. J. C. C. Gregor, "A survey on unmanned aerial and aquatic vehicle multi-hop networks: Wireless communications, evaluation tools and applications," vol. 119, pp. 43-65, 2018.

[11] B. L. R. Stojkoska and K. V. J. J. o. C. P. Trivodaliev, "A review of Internet of Things for smart home: Challenges and solutions," vol. 140, pp. 1454-1464, 2017.

[12] T. Rault, A. Bouabdallah, Y. Challal, F. J. P. Marin, and M. Computing, "A survey of energy-efficient context recognition systems using wearable sensors for healthcare applications," vol. 37, pp. 23-44, 2017.

[13] P. Rajpoot and P. J. W. P. C. Dwivedi, "Multiple parameter based energy balanced and optimized clustering for WSN to enhance the Lifetime using MADM Approaches," pp. 149, 2019.

[14] S. Hosseinirad, M. Alimohammadi, S. Basu, and A. J. I. T. A. B. Pouyan, "LEACH routing algorithm optimization through imperialist approach," vol. 27, no. 1, 2014.

[15] M. S. Karyakarte, R. G. Khanna, and A. S. G. Tavildar, "Energy Efficient Routing Strategy for Dynamic Wireless Sensor Networks," 2016. 


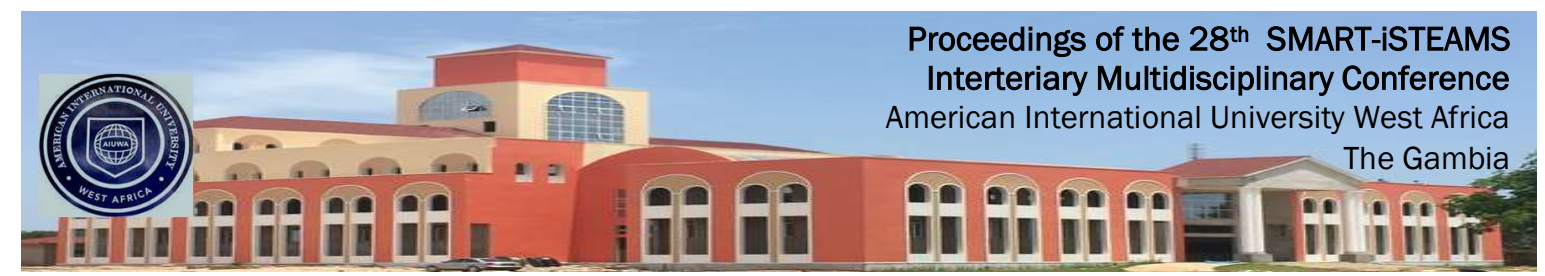

[16] A. R. Dhakne and P. Chatur, "A Comprehensive Survey on Intrusion Detection Systems in Wireless Sensor Network," in International Conference on Smart Trends for Information Technology and Computer Communications, 2016, pp. 541-549: Springer.

[17] T.-D. Nguyen, T. T. Thanh, L.-L. Nguyen, and H.-T. Huynh, "On the design of energy efficient environment monitoring station and data collection network based on ubiquitous wireless sensor networks," in The 2015 IEEE RIVF International Conference on Computing \& Communication Technologies-Research, Innovation, and Vision for Future (RIVF), 2015, pp. 163-168: IEEE.

[18] S.-c. Lee, G.-y. Lin, W.-r. Jih, C.-C. Huang, and J. Y.-j. Hsu, "Energy-Aware agents for detecting nonessential appliances," in International Conference on Principles and Practice of Multi-Agent Systems, 2010, pp. 475-486: Springer.

[19] S. K. Singh, M. Singh, D. J. I. J. o. A. N. Singh, and Application, "A survey of energyefficient hierarchical cluster-based routing in wireless sensor networks," vol. 2, no. 02, pp. 570-580, 2010.

[20] D. Medhi and K. Ramasamy, Network routing: algorithms, protocols, and architectures. Morgan Kaufmann, 2017.

[21] H. Li, Y. Liu, W. Chen, W. Jia, B. Li, and J. J. C. C. Xiong, "COCA: Constructing optimal clustering architecture to maximize sensor network lifetime," vol. 36, no. 3, pp. 256268, 2013.

[22] S. Ehsan, B. J. I. C. S. Hamdaoui, and Tutorials, "A survey on energy-efficient routing techniques with QoS assurances for wireless multimedia sensor networks," vol. 14, no. 2, pp. 265-278, 2012.

[23] S. Bandyopadhyay and E. J. Coyle, "An energy efficient hierarchical clustering algorithm for wireless sensor networks," in IEEE INFOCOM 2003. Twenty-second Annual Joint Conference of the IEEE Computer and Communications Societies (IEEE Cat. No. 03CH37428), 2003, vol. 3, pp. 1713-1723: IEEE.

[24] N. Sabor, S. Sasaki, M. Abo-Zahhad, S. M. J. W. C. Ahmed, and M. Computing, "A comprehensive survey on hierarchical-based routing protocols for mobile wireless sensor networks: review, taxonomy, and future directions," vol. 2017, 2017.

[25] X. J. s. Liu, "A survey on clustering routing protocols in wireless sensor networks," vol. 12, no. 8, pp. 11113-11153, 2012.

[26] J. Khan and H.-S. J. S. Cho, "A distributed data-gathering protocol using AUV in underwater sensor networks," vol. 15, no. 8, pp. 19331-19350, 2015.

[27] M. Ilyas and I. Mahgoub, Smart Dust: Sensor network applications, architecture and design. CRC press, 2018.

[28] T. Van Dam and K. Langendoen, "An adaptive energy-efficient MAC protocol for wireless sensor networks," in Proceedings of the 1st international conference on Embedded networked sensor systems, 2003, pp. 171-180: ACM.

[29] S. K. Singh, M. Singh, D. K. J. I. J. o. C. S. Singh, and E. Survey, "Routing protocols in wireless sensor networks-A survey," vol. 1, no. 2, pp. 63-83, 2010.

[30] X. J. I. S. J. Liu, "Atypical hierarchical routing protocols for wireless sensor networks: A review," vol. 15, no. 10, pp. 5372-5383, 2015. 


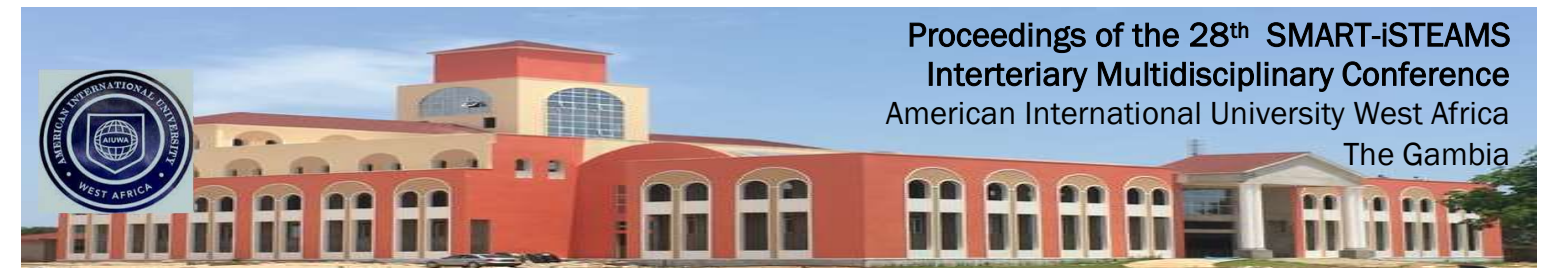

[31] A. Kinalis, S. Nikoletseas, D. Patroumpa, and J. J. I. f. Rolim, "Biased sink mobility with adaptive stop times for low latency data collection in sensor networks," vol. 15, pp. 5663, 2014.

[32] J. J. Lotf, M. Hosseinzadeh, and R. M. Alguliev, "Hierarchical routing in wireless sensor networks: a survey," in 2010 2nd international conference on computer engineering and technology, 2010, vol. 3, pp. V3-650-V3-654: IEEE.

[33] F. R. Dogar, T. Karagiannis, H. Ballani, and A. Rowstron, "Decentralized task-aware scheduling for data center networks," in ACM SIGCOMM Computer Communication Review, 2014, vol. 44, no. 4, pp. 431-442: ACM.

[34] N. B. Truong, G. M. Lee, and Y. Ghamri-Doudane, "Software defined networking-based vehicular adhoc network with fog computing," in 2015 IFIP/IEEE International Symposium on Integrated Network Management (IM), 2015, pp. 1202-1207: IEEE.

[35] K. A. Darabkh, S. Wala'a, M. Hawa, R. J. C. Saifan, and E. Engineering, "MT-CHR: A modified threshold-based cluster head replacement protocol for wireless sensor networks," vol. 72, pp. 926-938, 2018.

[36] A. A. Kharazian, K. Jamshidi, M. R. J. I. J. o. A. h. Khayyambashi, Sensor, and U. Computing, "Adaptive clustering in wireless sensor network: considering nodes with lowest-energy," vol. 3, no. 2, p. 1, 2012.

[37] G. Anastasi, M. Conti, M. Di Francesco, and A. J. A. h. n. Passarella, "Energy conservation in wireless sensor networks: A survey," vol. 7, no. 3, pp. 537-568, 2009.

[38] Z. Liu, Q. Zheng, L. Xue, and X. J. F. G. C. S. Guan, "A distributed energy-efficient clustering algorithm with improved coverage in wireless sensor networks," vol. 28, no. 5, pp. 780-790, 2012.

[39] R. Sheikhpour, S. Jabbehdari, A. J. I. J. o. A. S. Khadem-Zadeh, and Technology, "Comparison of energy efficient clustering protocols in heterogeneous wireless sensor networks," vol. 36, pp. 27-40, 2011.

[40] D. Izadi, J. Abawajy, and S. J. I. s. j. Ghanavati, "An alternative clustering scheme in WSN," vol. 15, no. 7, pp. 4148-4155, 2015.

[41] C. Wei, J. Yang, Y. Gao, and Z. Zhang, "Cluster-based routing protocols in wireless sensor networks: a survey," in Proceedings of 2011 International Conference on Computer Science and Network Technology, 2011, vol. 3, pp. 1659-1663: IEEE.

[42] M. Dhivya, M. Sundarambal, L. N. J. I. J. o. C. Anand, Network, and S. Sciences, "Energy efficient computation of data fusion in wireless sensor networks using cuckoo based particle approach (CBPA)," vol. 4, no. 04, p. 249, 2011.

[43] I. F. Akyildiz and I. H. J. A. h. n. Kasimoglu, "Wireless sensor and actor networks: research challenges," vol. 2, no. 4, pp. 351-367, 2004.

[44] A. Boulis, S. Ganeriwal, and M. B. J. A. h. n. Srivastava, "Aggregation in sensor networks: an energy-accuracy trade-off," vol. 1, no. 2-3, pp. 317-331, 2003.

[45] M. Cesana, F. Cuomo, and E. J. A. H. N. Ekici, "Routing in cognitive radio networks: Challenges and solutions," vol. 9, no. 3, pp. 228-248, 2011.

[46] M. Demmer and K. Fall, "DTLSR: delay tolerant routing for developing regions," in Proceedings of the 2007 workshop on Networked systems for developing regions, 2007, p. 5: ACM.

[47] N. Li, J. C. Hou, and L. J. I. T. o. W. C. Sha, "Design and analysis of an MST-based topology control algorithm," vol. 4, no. 3, pp. 1195-1206, 2005. 


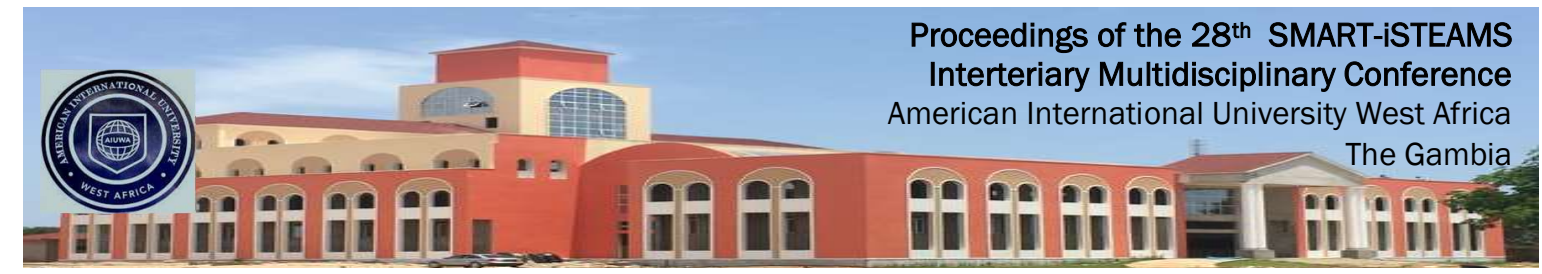

[48] C. Alippi, G. Anastasi, M. Di Francesco, M. J. I. I. Roveri, and M. Magazine, "Energy management in wireless sensor networks with energy-hungry sensors," vol. 12, no. 2 , pp. 16-23, 2009.

[49] A. A. Abbasi and M. J. C. c. Younis, "A survey on clustering algorithms for wireless sensor networks," vol. 30, no. 14-15, pp. 2826-2841, 2007.

[50] D. C. Hoang, P. Yadav, R. Kumar, and S. K. J. I. t. o. i. i. Panda, "Real-time implementation of a harmony search algorithm-based clustering protocol for energyefficient wireless sensor networks," vol. 10, no. 1, pp. 774-783, 2014.

[51] R. Ibrahim, Q.-D. Ho, and T. Le-Ngoc, "An energy-efficient and load-balancing clusterbased routing algorithm for csma-based wireless sensor networks," in 2013 IEEE 77th Vehicular Technology Conference (VTC Spring), 2013, pp. 1-5: IEEE.

[52] S. Tyagi, N. J. J. o. N. Kumar, and C. Applications, "A systematic review on clustering and routing techniques based upon LEACH protocol for wireless sensor networks," vol. 36, no. 2, pp. 623-645, 2013.

[53] S. Tanwar, N. Kumar, J. J. J. J. o. n. Rodrigues, and c. applications, "A systematic review on heterogeneous routing protocols for wireless sensor network," vol. 53, pp. 39-56, 2015.

[54] I. S. AIShawi, L. Yan, W. Pan, and B. Luo, "Lifetime enhancement in wireless sensor networks using fuzzy approach and A-star algorithm," 2012.

[55] J. N. Al-Karaki and A. E. J. I. w. c. Kamal, "Routing techniques in wireless sensor networks: a survey," vol. 11, no. 6, pp. 6-28, 2004.

[56] N. Xu, A. Huang, T. W. Hou, H. H. J. W. C. Chen, and M. Computing, "Coverage and connectivity guaranteed topology control algorithm for cluster-based wireless sensor networks," vol. 12, no. 1, pp. 23-32, 2012.

[57] J. H. Roh, M.-Y. Kim, and H. K. Moon, "An approach to designing lightweight security protocol on binary CDMA sensor networks," in 2009 International Conference on Ultra Modern Telecommunications \& Workshops, 2009, pp. 1-6: IEEE.

[58] J. Espina, T. Falck, A. Panousopoulou, L. Schmitt, O. Mülhens, and G.-Z. Yang, "Network topologies, communication protocols, and standards," in Body sensor networks: Springer, 2014, pp. 189-236.

[59] B. L. Titzer, D. K. Lee, and J. Palsberg, "Avrora: Scalable sensor network simulation with precise timing," in IPSN 2005. Fourth International Symposium on Information Processing in Sensor Networks, 2005., 2005, pp. 477-482: IEEE. 